\title{
The Symbolic Approach to Hybrid Systems
}

\author{
Thomas A. Henzinger \\ University of California, Berkeley
}

\begin{abstract}
A hybrid system is a dynamical system whose state has both a discrete component, which is updated in a sequence of steps, and a continuous component, which evolves over time. Hybrid systems are a useful modeling tool in a variety of situations, including the embedded (digital) control of physical (analog) plants, robotics, circuits, biology, and finance. We survey a computational approach to the verification and control of hybrid systems which is based on the symbolic discretization of continuous state changes. On the theoretical side, we classify infinite, hybrid state spaces as to which finite, discrete abstractions they admit. This classification enables us to apply concepts and results from concurrency theory, model checking, and game theory to hybrid systems. On the practical side, we discuss several options for implementing the symbolic approach to hybrid systems, and point to existing tool support.
\end{abstract}

